2017-01-01

\title{
Development of an immersive interface for robot teleoperation
}

\author{
Chen, J
}

http://hdl.handle.net/10026.1/15865

\subsection{7/978-3-319-64107-2_1}

Lecture Notes in Computer Science (including subseries Lecture Notes in Artificial Intelligence and Lecture Notes in Bioinformatics)

All content in PEARL is protected by copyright law. Author manuscripts are made available in accordance with publisher policies. Please cite only the published version using the details provided on the item record or document. In the absence of an open licence (e.g. Creative Commons), permissions for further reuse of content should be sought from the publisher or author. 


\title{
Development of an Immersive Interface for Robot Teleoperation
}

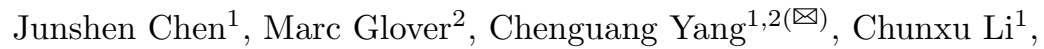 \\ Zhijun $\mathrm{Li}^{3}$, and Angelo Cangelosi ${ }^{2}$ \\ 1 Zienkiewicz Centre for Computational Engineering, \\ Swansea University, Swansea SA1 8EN, UK \\ cyang@theiet.org \\ 2 Center for Robotics and Neural Systems, Plymouth University, \\ Plymouth PL4 8AA, UK \\ 3 College of Automation Science and Engineering, \\ South China University of Technology, Guangzhou 510640, China
}

\begin{abstract}
In this paper, a novel interface of human-robot interaction has been developed to provide enhanced user experience for teleoperators. The interface has been implemented and tested on a Baxter robot platform and it can be easily adapted to other robot platforms. The main objective of this work is to provide a teleoperator immersive experience when controlling a telerobot arm by enabling the user to see and feel what the robot sees and feels from a first person point of view. This objective has been achieved by our designed interface integrating a haptic feedback device, a virtual reality headset, and an RGB-D camera. An operator can manipulate a robotic arm and receive force feedback information about interactions between the robot's grippers, as well as the robot's environment, whilst viewing the captured visual information of the robot's workspace, on the screen of the virtual reality headset. A servo motor driving platform has been designed as a new robot head to manipulate the camera on top of it, such that a teleoperator is able to control the pose of the camera in a natural manner via the wearable virtual reality headset. The orientation of the built-in inertial measurement unit (IMU) of the virtual reality headset is used to directly command the angles of the head platform on which the camera is mounted. The operator will have an immersive and in-depth experience when manipulating the robotic arm. Extensive tests with a variety of users have been carried out to evaluate the design in this work with quantified analysis.
\end{abstract}

Keywords: Teleoperation · Immersive virtual feedback $\cdot$ Human-robot interaction

\section{Introduction}

With the advance of automation technologies, robots have become increasingly prominent in our everyday lives [1]. Autonomous robots have become in com-

This work was supported in part by EPSRC grants EP/L026856/1 and EP/J004561/1 (BABEL) as well as Royal Society Newton Mobility Grant IE150858. 
mon use in industrial, as these robots are able to complete high precision tasks with minimal completion time, especially within hazard environments. However, specialised tasks that require extensive interactions with uncertain environment, e.g., it is not suitable for fully-autonomous robots to complete medical operation. A tele-controlled robot with human operator may be desired for these tasks [2], i.e., teleoperation. Works demonstrated in [3] showed how stereo vision contributes to improve the performance of applications in mobile robot teleguide. [4] presents a non-contacting vision-based method of robot teleoperation which improves accuracy of object gripping tasks. Teleoperation has progressed to the point, where human operators can easily control a multi-jointed robotic arm [5-8], via a haptic feedback device. As introduced in [9], with haptic force feedback, a pilot can have a natural representation when approaching obstacles. Force feedback was also added in [10] to aid camera system avoiding obstacle in mobile robot teleoperation. It is natural to combine haptic information with visual information for coordinated information feedback. However, conventional means of visual feedback using a monitor do not provides to an operator an immersive user experience. To enhance the telepresence, in this work, an immersive visual feedback device Oculus Rift DK2 is employed, together with a Kinect v2 RGB-D imaging device, and haptic device Geomagic Touch, to teleoperate a Baxter robot arm (Fig. 1).

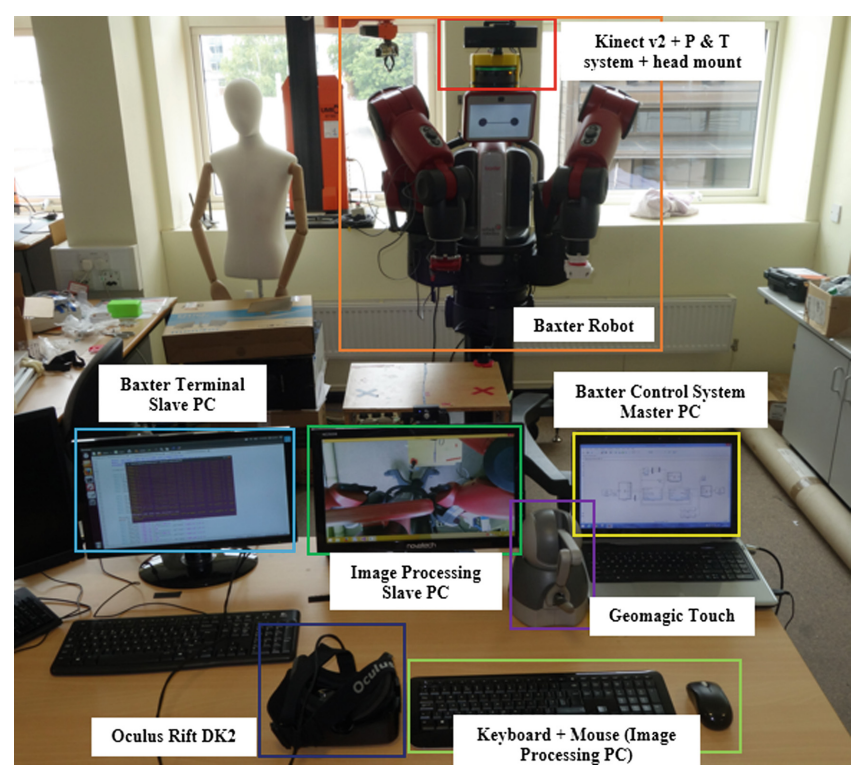

Fig. 1. Illustration of the teleoperation setup, with each key component labelled, including a Kinect head mount system, a Baxter robot, a Geomagic Touch and an Oculus Rift DK2. [photo taken at Plymouth University] 
Haptic feedback devices which give an operator force feedback from a telerobot have been utilised by numerous researchers $[11,12]$. In this work, using IR sensor at the cuff of Baxter's end effector (Fig. 2), the haptic feedback information is provided as the distance between an object/surface and the baxter's gripper. This haptic force feedback is also bonded with two other customised force feedbacks, tactile force, and restoration force. Tactile force is derived from a force sensing resistor (FSR) mounted onto one of the pincers of Baxter's end effector gripper (referred to as the FSR pincer, Fig. 2), which allows an operator to sense the firmness of objects picked up with the gripper. Restoration force tends to return the haptic joystick back to the preset original pose, after an operator has used the haptic joystick to change the position of the robot's gripper's pincers. Force feedback is presented to an operator via a Geomagic Touch haptic feedback joystick (Fig. 1), which is also used by the operator to control the position of Baxter's end effector.

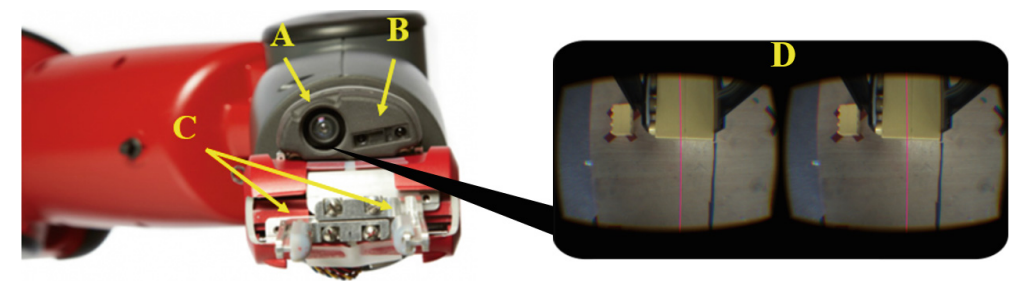

Fig. 2. Illustration of one of the Baxter robot's end effectors. A: the cuff camera; B: the IR range sensor; C: active, gripper attachment [20]; D: VR headset capture of the operators view of the robot workspace, captured by the cuff camera

Immersive visual feedback technique which provides an operator a sense of telepresence has been implemented in many research projects [13,14]. In [15], a panoramic display system was used for improving teleoperation performance of a mining robot. An virtual reality (VR) headset, Oculus Rift, was used in [16] to control a simulated robot in a combat scenario. An intuitive $3 \mathrm{D}$ interface was designed to allow operator immersed in a virtual environment while teleoperating [17]. Rather than using the conventional interface composed of a monitor and a keyboard [18], immersive visual feedback technique can give operators a feeling that they are controlling robot as sitting inside robot. In this paper, the output image stream after image processing is displayed on the Oculus Rift DK2 headset. The operator is be able to manipulate the gaze of the Kinect camera's field of view (via a servo pan and tilt kit), as they move their head in a manner which is naturally similar, towards a certain direction for a better view. The IMU unit built into the Oculus Rift headset tracks the operator's head motion, and this subsequently turns the developed pan and tilt servo platform on which the Kinect camera is mounted. The Kinect pan and tilt servo platform is mounted onto the head of Baxter, and enables an operator to feel as though they are looking at the robot's workspace from a static position, in place of the robot. 
In order for the operator to control the gaze of visual feedback camera, i.e., Kinect v2, a pan and tilt servo mount has been designed and manufactured, and fixed on top of Baxter's head. This also could be beneficial to other research carried out on the Baxter robot platform in the future. Baxter is a robust, reliable research robot platform which has only been available for nearly three years, and research already conducted on this platform has shown very promising and interesting results, this project will be the first of its kind to implement a system which combines haptic, immersive visual feedback on this platform. Extensive tests have been carried out, based on our previous work [19], to compare the user experiences of non-immersive and immersive teleoperation interfaces and their performances.

\section{System Communications}

Commands/data are sent/received via User Datagram Protocol (UDP) sockets, over a local wireless network as shown in Fig. 3, between the slave (Baxter terminal) computer and the Baxter robot. Two additional computers are employed, namely, a master computer, which is connected to the Geomagic Touch haptic joystick and controls the position of the Baxter's end effector, and a slave computer, which captures and processes images from the Kinect v2 device and from the cuff camera of Baxter's right arm (Fig. 2), as well as rendering, and serving these images to the Oculus Rift headset. This computer also sends the orientation of the Oculus Rift to the Arduino microcontroller which controls the pose of the head platform. All communications between the single master, the two slave computers, the Arduino microcontroller system, the and Baxter robot, are transmitted over the local wireless network. A Baxter control system model, interfaces with the Geomagic Touch haptic feedback controller, to apply force

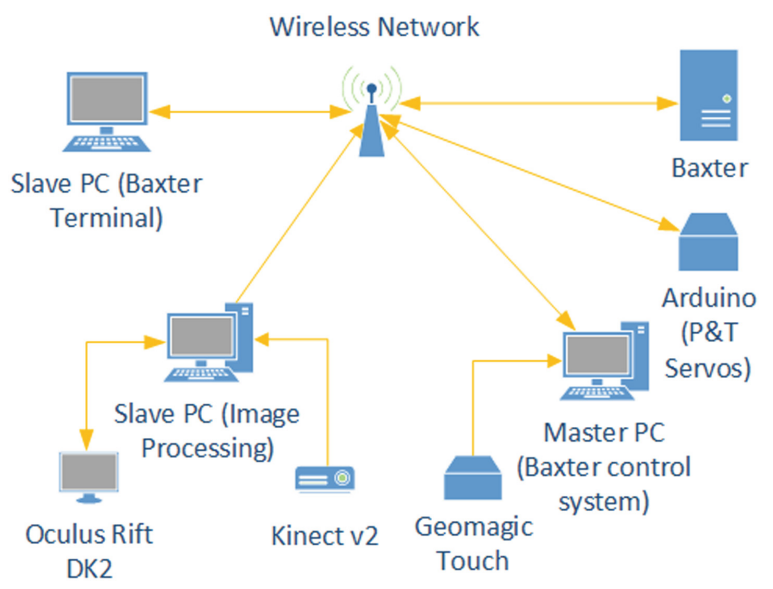

Fig. 3. Network diagram, representing each PC/terminal as a node on the local wireless network 
feedback to the operator via the controller, and sets the position of the Baxter's end effector from the position of the haptic feedback controller's end effector (via the slave/Baxter terminal computer). This model is executed on the master computer. In order to control the orientation and position of the Baxter's end effector, the master computer must send the end effector orientation and position data to the slave (Baxter terminal) computer, which is subsequently sent to the Baxter robot. A Baxter control script written in Python is running on the slave computer (Baxter terminal), listening for commands from the master computer, interpreting them, and then sending the commands to the Baxter (sent as ROS commands). The overall communications system is shown in Fig. 3.

\section{Haptic Feedback and Teleoperation Control}

\subsection{Position and Orientation Control}

The position of the Baxter's end effector is determined by the position of the first three joints of the Geomagic Touch haptic feedback controller. A Simulink program developed in our previous work [5] is used to interface between the master computer and the Geomagic Touch controller. The Baxter's end effector position commanded by the Geomagic Touch, must first be modified to match the Baxter's frame as detailed in [5]. This position is then sent to the Baxter robot, via the slave Baxter terminal PC. To control the orientation of the Baxter's end effector, the transformation matrix, which include the rotation and translation of the 4th, 5th and 6th joints of the Geomagic Touch controller is used to calculate the Euler angles of the roll, pitch, and yaw of the Baxter's end effector.

\subsection{Haptic Feedback}

Tactile Force. Using IR sensor at the cuff of Baxter's end effector, the haptic feedback information is provided as the distance between an object and the baxter's gripper. The operator receives haptic feedback continuously while controlling the position and orientation of the Baxter's end effector. The haptic joystick provides to the operator feedback information about the workspace environment, which can be noticed both consciously and subconsciously by the operator. Operator using only a joystick is not able to receive tactile feedback when grasping an object with the robot arm's gripper. Tactile feedback whilst grasping an object is important for enhanced user experience of natural feeling and delicate manipulation of soft objects. Therefore, a simple one dimensional force sensor is added one of the pincer pads, on the end effector's gripper.

The operator can change the position of both pincers of the robot arm gripper's end effector, by pressing the second button on the joystick stylus (Fig. 4a). Changing the position of the feedback joystick's first joint will now open or close the gripper (Fig. 4c, d), with a resolution of 256 divisions (8-bit unsigned). The position of the robot arm and the end effector will also be fixed, so that only the gripper's pincers may move. The operator only feels force feedback through 
the haptic joystick when the FSR has some force applied to it, and when the operator has pressed the second stylus button to fix the position of the robot arm, in order to change the position of the gripper's pincers. The operator can now feel the firmness/consistency of an object, which is in-between the gripper's pincers; through force feedback. The force exerted by the gripper's pincers on the object is however constant, therefore the operator does not receive true (natural feeling) haptic information about the firmness/consistency of an object.

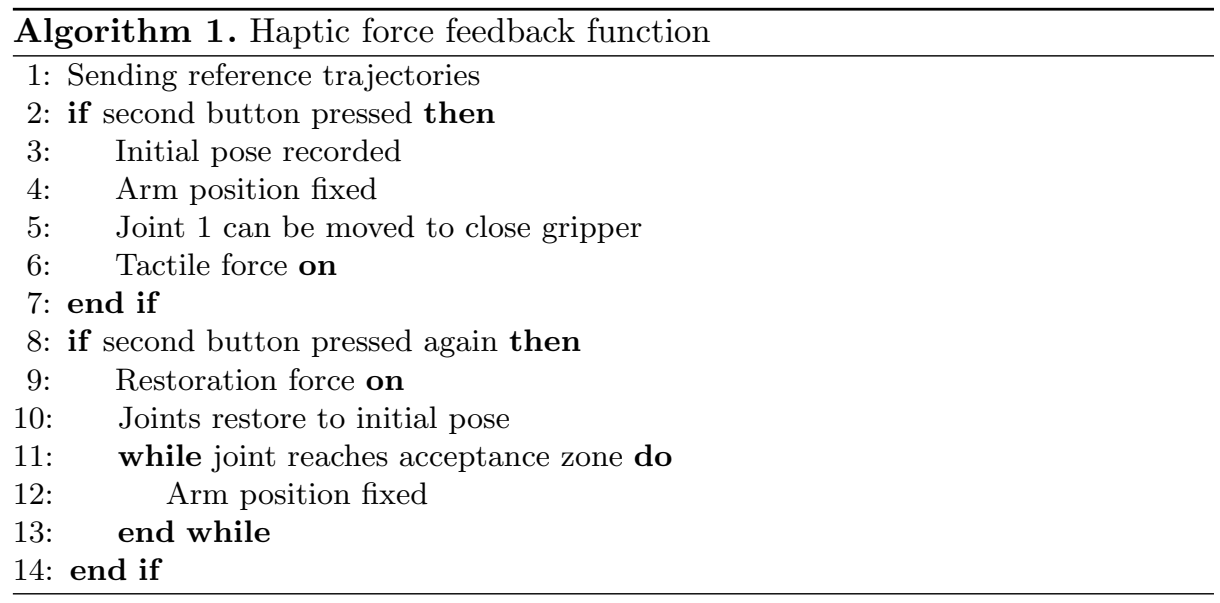

Restoration Force. When the operator has changed the position of the gripper's pincers, the operator then presses the second stylus button to release the position of the robot arm. However, since the angle of the haptic joystick's joints has changed (Fig. 4c), the position of the robot arm will stay in the fixed position (Fig. 4f), until the angles of the haptic joystick's joints match the angles of the haptic joystick's joints when the second stylus button was initially pressed (Fig. $4 \mathrm{~g}$ ); an acceptance zone of roughly $\pm 5 \%$ of the initial haptic joystick's joint angles is used.

To force the angles of the haptic joystick's joints to go back to their respective angles when the second stylus button was initially pressed (Fig. 4e), the angle of each of the haptic joystick's joint at the time of the initial button pressed is respectively fed into a separate PD controller as the desired angle, and the current haptic joystick's joint angles as the process variable; the output of each $\mathrm{PD}$ controller is used to create a 3 dimensional joint torque vector, which is then supplied to the Geomagic Touch joystick and given to the operator as force feedback (which exhibits spring-like behaviour, Fig. 4e):

$$
T=K_{p}\left(P_{S P}-P_{P V}\right)+K_{d}\left(\dot{P}_{S P}-\dot{P}_{P V}\right)
$$

where haptic feedback controller joint torques $T=\left[T_{J 1} T_{J 2} T_{J 3}\right]^{T} \in R^{3 \times 1}$, $K_{p}$ and $K_{d}$ are properly chosen gains, $P_{S P}$ is the preset point, and $P_{P V}$ is 


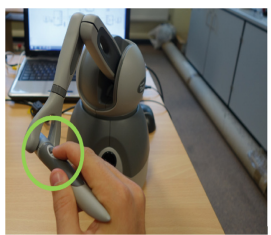

(a) Second button pressed, initial joint tions are fixed positions recorded

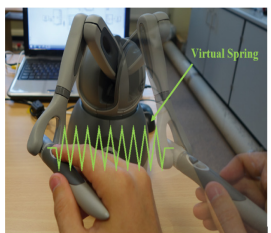

(e) Press

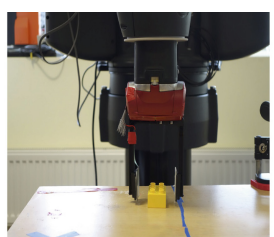

(b) Arm joints posi-

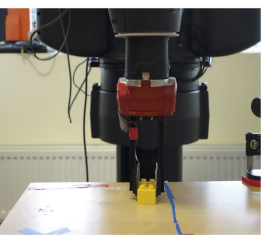

(f) Arm

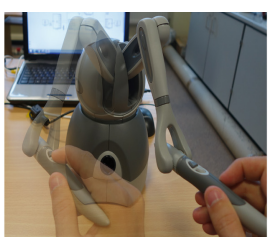

(c) Moving joint 1, to close grippers

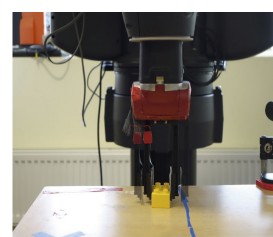

(d) Gripper closed (e) Press the (f) Arm pose is (g) Geomagic joints (h) Arm joints re-
button again, joints fixed until Geo- have reached the leased, normal posiback to initial pose magic joints have acceptance zone, tion control resumes (controllers enabled) each reached the haptic feedback acceptance zone stops (controllers disabled)

Fig. 4. Illustrates the function of the haptic feedback PD controllers, returning the Geomagic joints to their initial angles, after the operator grasps an object with the end effector gripper. The light grey button nearest the operators hand is referred to as the second button, and the dark grey button is referred to as the first button, respectively

the process variable point. These PD controllers are disabled when each of the joystick's joints have reached their respective acceptance zone.

Changing Perspective. The operator has the option to display the Kinect colour images, or camera images captured from the end effector camera of the robot arm (Fig. 2). When the operator switches views to the end effector camera, a gain of $K$ is applied to all end effector position movements transmitted to the Baxter robot (Eq. 2):

$$
P_{p m}=P_{i n i}+K\left(P_{c u r}-P_{i n i}\right)
$$

where $P_{p m}$ is the end effector position, $P_{i n i}$ is the initial end effector position, in precision mode, $P_{\text {cur }}$ is the end effector position calculated by the Geomagic Touch controller block, using standard position mapping [5]. Since the position mapping between the Geomagic and robot arm has changed, the angles of the Geomagic's joints are returned to their respective initial angles. 


\section{Visual Feedback System}

\subsection{Mounting Kinect V2 on Baxter}

The head platform was purposely designed to fit directly onto the head of Baxter in SolidWorks, and 3D printed (Fig. 5). This head platform does not require any modifications to be made to the existing Baxter robot, since the platform attaches directly to the head of Baxter (as shown in Fig. 6a). The legs of the head platform allow the platform to be fixed around the head of the Baxter robot, whilst not interfering with the field of view of the robot's sonar ring (Fig. 6a).

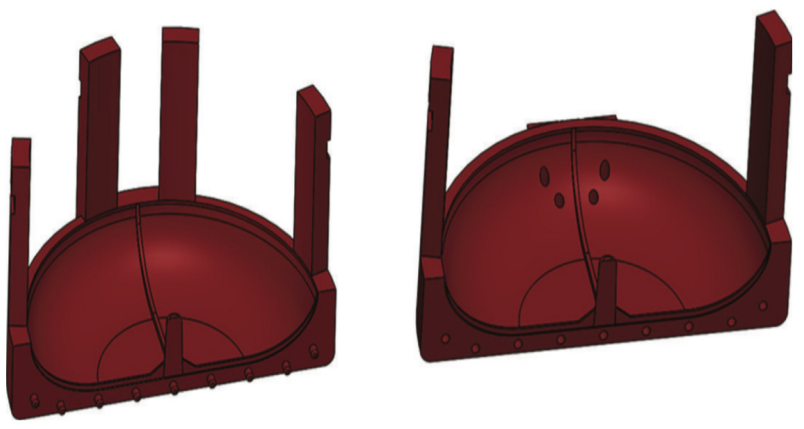

Fig. 5. The two halves of the Kinect - Baxter head mounting base; back half (left, and front half (right). The cross section shows the profile of the Baxter head cap, and central peg. The legs (with notches), fix the head mounting base, to the head of Baxter

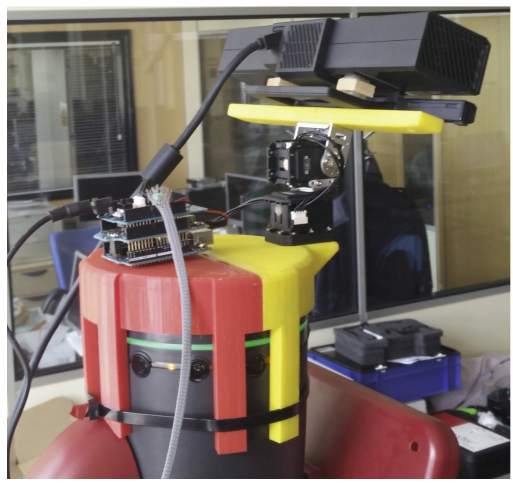

(a)

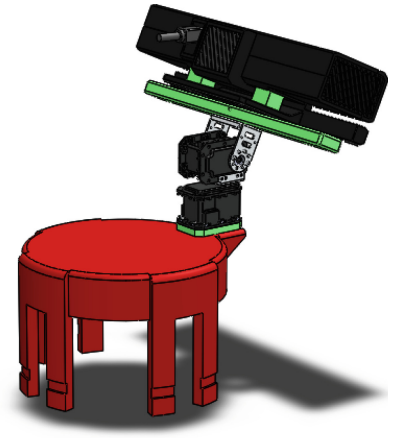

(b)

Fig. 6. (a) The $3 \mathrm{D}$ printed head platform attached directly to the head cap of the Baxter robot (grey ABS dish, above green LED ring); the platform's six legs fit around the head of the Baxter. (b) The complete pan and tilt servoing platform with Kinect amounted; 3D printed support bar (green) attaches the Kinect to the tilt servo bracket, MDF pan servo spacer (green) mounts the servo assembly to the Baxter head mount. (Color figure online) 
Small notches in each of the platform's legs (Fig. 6b), guide a zip tie which is used to fix the head platform laterally to the robot. Two Dynamixel MX28R servo, one tilt servo and one pan servo, was directly attached to the edge of the head platform. It can hold the Kinect v2 with a 3D printed support bar attached to the tilt servo. This gives us the view from Baxter's head.

\subsection{Viewing the Robot Workspace}

Controlling Kinect's View. We used an Arduino Microcontroller Development board to control the both pan and tilt positions of MX-28R servos. Servo data packets containing the desired servo setting position are output via the Arduino's USART peripheral, to a TTL to RS485 converter, in order to convert TTL logic level signals to RS485 levels (MX-28R utilise the RS485 communications protocol). The Arduino is connected to a Wi-Fi shield, from which the Arduino can receive UDP packets, which contain encoded servo position strings, constructed as follows: Servo ID, separating character, desired servo position, and finally an end-of-file character. The broadcast string "S1,1023 $\backslash 0$ ", will set servo ID 1 (pan servo), to position $1023\left(90^{\circ}\right)$. The Arduino decodes the received strings, and sets the pan and tilt servo positions appropriately. Using the image processing slave PC (Fig. 3), pitch and yaw angles of the Oculus Rift are acquired, encoded into a position string, and subsequently sent as an UDP packet via WiFi to the Arduino Wi-Fi shield. Pitch and yaw angles are given as Euler angles by the Oculus Rift Software Development Kit (SDK). So that the Kinect sensor will follow the operator's head motion when they turn or tilt their head.

Rendering on Oculus Rift. To give the most immersive experience possible to the operator, the output of the Kinect v2s colour camera (the view from Baxter), is displayed on the screen of the Oculus Rift DK2. This gives the operator the sensation that they are looking at the workspace through the eyes of Baxter. To do this, the current colour and depth frames are captured from the Kinect v2. A distortion was applied to original image (as shown in Fig. 7) to cancel Oculus Rift's lenses' distortion. A flat image of Oculus's capture will present to operator as final image (unless stereoscopic effects are used to create a 3 dimensional view of the environment). The captured $1920 \times 1080$ pixels colour image from the Kinect v2 is presented to each eye of the Oculus Rift; the Oculus SDK then internally rescales the size of each image to match the resolution of each eye.

A Natural View of the Workspace. Oculus Rift DK2 features a built-in 9-axis IMU (Inertial Measurement Unit); a 6-axis MEMS gyroscope and accelerometer (Invensense MPU-6500), and a 3-axis MEMS magnetometer (undisclosed manufacturer and model). The Oculus SDK can be used to extract the Euler angles of the Oculus Rift, from the IMU; the IMU applies digital low pass filtering to the output gyroscope and accelerometer on-chip, the SDK reads these outputs and converts the accelerometer and gyroscope data from Quaternion notation to Euler angle notation. Euler showed that any rotation in a 3 


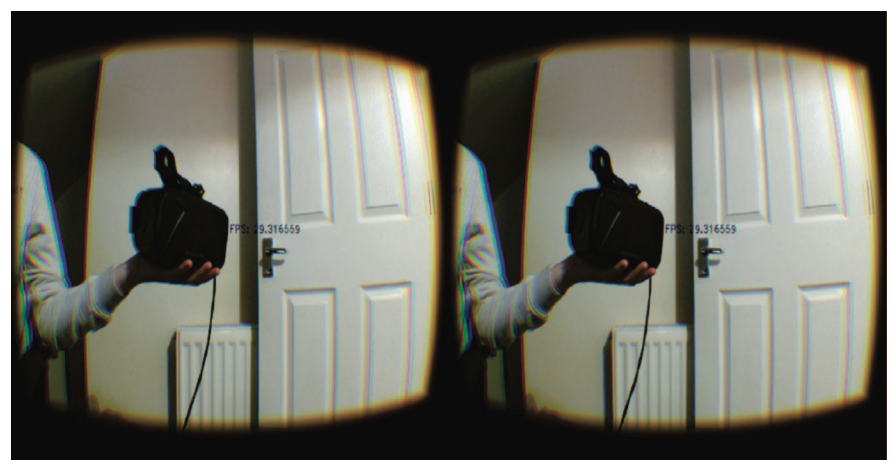

Fig. 7. Radial distortion is added to the input image before it is displayed on the Oculus Rift, to compensate for radial distortion due to the headset's lenses

dimensional space, can be described as 3 rotation about each axis ( $\mathrm{x}, \mathrm{y}$, and $\mathrm{z})$. Therefore, obtaining the Euler angles of the Oculus Rift via the Oculus SDK, represents the orientation of the operator's head as roll (about the $\mathrm{z}$ axis), pitch (about the $\mathrm{x}$ axis), and yaw (about the $\mathrm{y}$ axis). However, the orientation of the Kinect v2 can only be altered for the yaw and pitch (pan and tilt servos), therefore the roll in this case is fixed; this extra degree of freedom could be added later by mounting the current Kinect pan tilt servo assembly, on top of a servo which will control the roll of the Kinect v2. The obtained Euler angles of the Oculus's pitch and yaw, are given in radians; these are converted into degrees to simplify the process of converting the pitch and yaw angles into the tilt and pan servo positions respectively. The conversion is given as follows:

$$
R_{s}=\frac{R_{O}}{R_{a}}
$$

where $R_{s}$ is the position of the tilt or pan of servo, $R_{O}$ is the pitch or yaw angle of the Oculus and $R_{a}=0.088^{\circ}$ is the angular resolution of servo. The clockwise rotations have been set as a positive rotation.

Changing Perspectives. During system testing, it is difficult for the operator to gain a sense of depth within Baxter's workspace; this is due to a significant distance between the Kinect's camera and the target object; when controlling Baxter to complete a pick and place operation. Due to the lack of the operator's sense of depth, a method was devised to change the images rendered of the Oculus Rift's display from the Kinect's colour video stream, to the camera built into the cuff of Baxter's right arm (as shown in Fig. 8). 


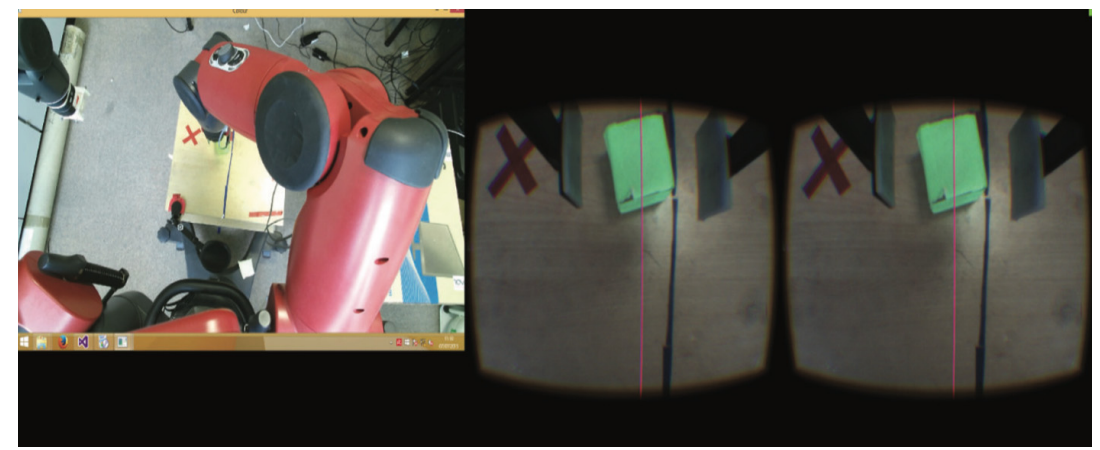

Fig. 8. Monitor outputs: left image is the image captured by Kinect on the top of Baxter, and the two distorted images on the right, are the right arm, cuff camera. A pink line represents the centre of the frame to make aligning the gripper with objects easier. (Color figure online)

\section{$5 \quad$ Test and Evaluation}

\subsection{Testing Scenario}

Twelve subjects were asked to pick up one Lego block each time and stack it on top of one another. Three different settings of the teleoperation system were tested: VR system plus force feedback on the haptic device; VR system only (without force feedback); and on-screen visual feedback with keyboard control of Kinect's position plus force feedback on the haptic device. The gain $K$ for the force feedback in Eq. 2 was set at 0.25 due to the designer's experience.

Number of collisions that the end-effector collided with the table was recorded while operator performing each task. The result was shown in Fig. 9. All the operators were asked to fill in a questionnaire after they completed all three tasks, the questionnaire is attached in Appendix A.

\section{End Effector Table Collisions}

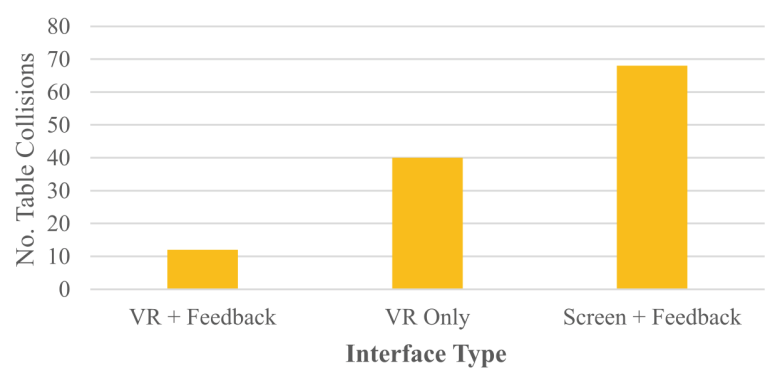

Fig. 9. Total number of collisions that the end-effector collided with the table while twelve operators performing each task. 


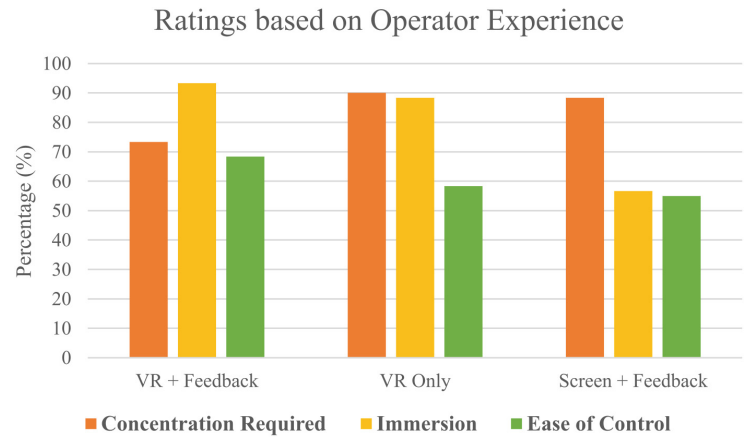

Fig. 10. Average user experience ratings of each system setting, including concentration required, immersion and ease of control.

It is illustrated from Fig. 9 that there is a relationship between the level of the immersion experienced by the operator and the collision times of robot's end effector. Since the number of the collision times increased significantly when the operators' immersion level decreased. The sensation of viewing the robot workspace in a way which is close to the natural humans view (as well as control of view), could be considered as the cause of this result.

The ratings of the three different teleoperation experiences is shown in Fig. 10. The least concentration level during teleoperation was required when both VR and force feedback applied. The level of immersion decreased dramatically between VR systems and screen visual feedback system. And it also became harder for the users to complete the task while the level of immersion decreased.

\section{Conclusion}

A novel human-robot interaction interface for teleoperation has been developed in this paper. The interface integrated both visual feedback and haptic feedback technologies to provide immersive user experience. The visual feedback is provided by a vision system mounted on a custom-built head platform, which is naturally controlled to track the head's motion of human operator. The designed interface provides operator an immersive sensation of viewing from robot's perspective, aiding operator in concentrating on completing a given task. In the meantime, operator is able to manipulate the position of the Kinect device in a natural way by moving his/her head. It can reduce the cognitive load on user while performing teleoperation. User experience investigation results show that the invited testers preferred using our designed interface to view visual feedback of the robot workspace and control the field of view of visual feedback, rather than using the conventional interface composed of a monitor and a keyboard. The use of virtual reality based interfaces in teleoperation projects is a solution which may become more apparent in the near future, since these interfaces offer immersive platforms for visual feedback to the operator, that can only be experienced on these types of devices. 


\section{Appendix}

\section{A Participant Questionnaire}

\section{Baxter Teleoperation - Questionnaire}

\begin{tabular}{|c|c|c|c|}
\hline \multirow{2}{*}{\multicolumn{4}{|c|}{$\begin{array}{l}\text { Gender: } \\
\text { Q1. }\end{array}$}} \\
\hline & & & \\
\hline \multicolumn{4}{|c|}{ Have you controlled any kind of robot before? } \\
\hline \multicolumn{4}{|c|}{ If yes, please give details: } \\
\hline \multicolumn{4}{|l|}{ Q2. } \\
\hline \multicolumn{4}{|c|}{ Have you used a virtual reality headset before? } \\
\hline \multicolumn{4}{|c|}{ If yes, please give details: } \\
\hline \multicolumn{4}{|c|}{$\begin{array}{l}\text { After completing the series of simple pick and place tasks, please complete the following } \\
\text { questions, by either highlighting, or circling your choices; } 0 \text { very poor, } 5 \text { excellent: }\end{array}$} \\
\hline & $\begin{array}{l}\text { Screen + } \\
\text { Keyboard }\end{array}$ & VR Only & VR + Haptic Feedback \\
\hline $\begin{array}{l}\text { System } \\
\text { Responsiveness }\end{array}$ & 012345 & 012345 & 012345 \\
\hline $\begin{array}{l}\text { Concentration } \\
\text { Required }\end{array}$ & 012345 & 012345 & 012345 \\
\hline $\begin{array}{l}\text { Immersive } \\
\text { Experience }\end{array}$ & 012345 & 012345 & 012345 \\
\hline Ease of Control & 012345 & 012345 & 012345 \\
\hline Task Difficulty & 012345 & 012345 & 012345 \\
\hline $\begin{array}{l}\text { Time to } \\
\text { complete task }\end{array}$ & 012345 & 012345 & 012345 \\
\hline Task Completed & Yes No & Yes No & Yes No \\
\hline \multicolumn{4}{|l|}{ Q3. } \\
\hline \multicolumn{4}{|c|}{$\begin{array}{l}\text { In terms of how immersive your experience was, how did controlling the robot while looking } \\
\text { at the PC monitor, compare with controlling the robot while looking through the virtual } \\
\text { reality headset? Which did you prefer? Please give reason(s) why: }\end{array}$} \\
\hline \multicolumn{4}{|l|}{ Q4. } \\
\hline \multicolumn{4}{|c|}{$\begin{array}{l}\text { Did you face any challenges when using the Kinect camera or the cuff camera? Was the } \\
\text { ability to switch between cameras helpful? }\end{array}$} \\
\hline \multicolumn{4}{|c|}{ Q5. } \\
\hline \multicolumn{4}{|c|}{$\begin{array}{l}\text { Was the received haptic feedback helpful? Was the force feedback you received what you } \\
\text { were expecting? Please give details: }\end{array}$} \\
\hline \multicolumn{4}{|l|}{ Q6. } \\
\hline Any suggestions & the VR + Hap & dback system & improved? \\
\hline
\end{tabular}




\section{References}

1. Billard, A., Calinon, S., Dillmann, R., Schaal, S.: Robot programming by demonstration. In: Siciliano, B., Khatib, O. (eds.) Springer Handbook of Robotics, pp. 1371-1394. Springer, Heidelberg (2008)

2. Sarmah, A., Gulhane, U.: Surgical robot teleoperated laparoscopic grasper with haptics feedback system. In: 2010 International Conference on Emerging Trends in Robotics and Communication Technologies, pp. 288-291, December 2010

3. Livatino, S., Muscato, G., Privitera, F.: Stereo viewing and virtual reality technologies in mobile robot teleguide. IEEE Trans. Robot. 25, 1343-1355 (2009)

4. Kofman, J., Wu, X., Luu, T.J., Verma, S.: Teleoperation of a robot manipulator using a vision-based human-robot interface. IEEE Trans. Industr. Electron. 52, 1206-1219 (2005)

5. Ju, Z., Yang, C., Li, Z., Cheng, L., Ma, H.: Teleoperation of humanoid Baxter robot using haptic feedback. In: 2014 International Conference on Multisensor Fusion and Information Integration for Intelligent Systems, pp. 1-6, September 2014

6. Reddivari, H., Yang, C., Ju, Z., Liang, P., Li, Z., Xu, B.: Teleoperation control of Baxter robot using body motion tracking. In: 2014 International Conference on Multisensor Fusion and Information Integration for Intelligent Systems, pp. 1-6, September 2014

7. Wang, X., Yang, C., Ma, H.: Automatic obstacle avoidance using redundancy for shared controlled telerobot manipulator. In: The 5th Annual IEEE International Conference on Cyber Technology in Automation, Control and Intelligent Systems, pp. 1338-1343, 8-12 June 2015

8. Liang, P., Yang, C., Li, Z., Li, R.: Writing skills transfer from human to robot using stiffness extracted from sEMG. In: The 5th Annual IEEE International Conference on Cyber Technology in Automation, Control and Intelligent Systems, pp. 19-24, 8-12 June 2015

9. Hou, X., Mahony, R.: Dynamic kinesthetic boundary for haptic teleoperation of vtol aerial robots in complex environments. IEEE Trans. Syst. Man Cybern. Syst. 46, 694-705 (2016)

10. Cho, S.K., Jin, H.Z., Lee, J.M., Yao, B.: Teleoperation of a mobile robot using a force-reflection joystick with sensing mechanism of rotating magnetic field. IEEE/ASME Trans. Mechatron. 15, 17-26 (2010)

11. Romano, J., Hsiao, K., Niemeyer, G., Chitta, S., Kuchenbecker, K.: Humaninspired robotic grasp control with tactile sensing. IEEE Trans. Robot. 27, 1-10 (2011)

12. Lee, S., Sukhatme, G., Kim, G.J., Park, C.M.: Haptic teleoperation of a mobile robot: a user study. Presence 14, 345-365 (2005)

13. Tachi, S., Komoriya, K., Sawada, K., Nishiyama, T., Itoko, T., Kobayashi, M., Inoue, K.: Telexistence cockpit for humanoid robot control. Adv. Robot. 17(3), 199-217 (2003)

14. Kim, D.Y., Lee, M.S., Choi, S.H., Koo, K.-J., Hwang, I., Kim, Y.J.: An immersive telepresence platform based on distributed architecture. In: 2013 International Conference on ICT Convergence, pp. 465-467, October 2013

15. James, C.A., Bednarz, T.P., Haustein, K., Alem, L., Caris, C., Castleden, A.: Teleoperation of a mobile mining robot using a panoramic display: an exploration of operators sense of presence. In: 2011 IEEE International Conference on Automation Science and Engineering, pp. 279-284, August 2011 
16. Conn, M.A., Sharma, S.: Immersive telerobotics using the oculus rift and the 5DT ultra data glove. In: 2016 International Conference on Collaboration Technologies and Systems, pp. 387-391, October 2016

17. Regenbrecht, J., Tavakkoli, A., Loffredo, D.: A robust and intuitive 3d interface for teleoperation of autonomous robotic agents through immersive virtual reality environments. In: 2017 IEEE Symposium on 3D User Interfaces, pp. 199-200, March 2017

18. Dünser, A., Lochner, M., Engelke, U., Fernández, D.R.: Visual and manual control for human-robot teleoperation. IEEE Comput. Graph. Appl. 35, 22-32 (2015)

19. Chen, J., Glover, M., Li, C., Yang, C.: Development of a user experience enhanced teleoperation approach. In: 2016 International Conference on Advanced Robotics and Mechatronics, pp. 171-177, August 2016

20. Rethink Robotics, May 2014. http://sdk.rethinkrobotics.com/mediawiki-1.22. 2/images/thumb/4/48/Hand_sensors.png/425px-Hand_sensors.png. Accessed 01 July 2015 